\title{
A parabolic mirror-based proximally actuated photoacoustic endoscope
}

Chiye Li, Joon-Mo Yang, Ruimin Chen, Konstantin Maslov, Qifa Zhou, et al.

Chiye Li, Joon-Mo Yang, Ruimin Chen, Konstantin Maslov, Qifa Zhou, K. Kirk Shung, Lihong V. Wang, "A parabolic mirror-based proximally actuated photoacoustic endoscope," Proc. SPIE 8581, Photons Plus Ultrasound:

Imaging and Sensing 2013, 858148 (4 March 2013); doi: 10.1117/12.2005494

SPIE. Event: SPIE BiOS, 2013, San Francisco, California, United States 


\title{
A parabolic mirror-based proximally actuated photoacoustic endoscope
}

\author{
Chiye $\mathrm{Li}^{1,3}$, Joon-Mo Yang ${ }^{1,3}$, Ruimin Chen ${ }^{2}$, Konstantin Maslov ${ }^{1}$, Qifa Zhou ${ }^{2}, \mathrm{~K}$. Kirk Shung ${ }^{2}$, \\ and Lihong V. Wang ${ }^{1, *}$ \\ ${ }^{1}$ Optical Imaging Laboratory, Department of Biomedical Engineering, Washington University in St. \\ Louis, One Brookings Drive, Campus Box 1097, St. Louis, Missouri, 63130, USA \\ ${ }^{2}$ Ultrasonic Transducer Resource Center, Department of Biomedical Engineering, University of \\ Southern California, 1042 Downey Way, University Park, DRB 130, Los Angeles, CA 90089, USA
}

\begin{abstract}
We have developed a new photoacoustic endoscopic probe specifically designed for human urogenital imaging. The endoscopic probe uses a parabolic mirror-based mechanical scanning mechanism, providing an angular field of view of $270^{\circ}$. And it has a rigid, dome shaped end section for smooth cavity introduction. Here we introduce the new endoscope's design and imaging principle, and present experimental results validating its in vivo imaging ability.
\end{abstract}

Keywords: Photoacoustic endoscopy, human urogenital imaging, cancer diagnosis, functional imaging.

\section{INTRODUCTION}

Cancer is an emerging public health problem as people live longer and the population increases. As strategies to this problem, early detection and monitoring during therapy are important parts of cancer control. Considering the high false negative rate of current cervical cancer screening, new cost-effective and more reliable diagnostic methods are necessary to complement current screening methods. For imaging tumors in deep-seated organs, developments of tomographic endoscopic techniques are important because such techniques can provide direct visions of tumors and facilitate accurate diagnosis. Although traditional endoscopic ultrasound technique is widely used for human urogenital imaging, they do not sufficiently provide important pathophysiological information.

Now, photoacoustic endoscopy (PAE) $)^{1-4}$ embodying photoacoustic tomography (PAT) $)^{5,6}$ in a small probe is emerging as a new, minimally invasive imaging tool for diagnosing diseases in internal organs, such as gastrointestinal tracts $1,3,4$, ${ }^{7}$ and urogenital systems ${ }^{8-11}$. Many neoplasms are associated with angiogenesis and show abnormal metabolic rates ${ }^{12}$. Based on the strong spectroscopic, functional, and angiographic imaging capability of PAT ${ }^{5,6,12-15}$, PAE can be used for diagnosing many urogenital system diseases such as endometrial cancer and prostate cancer. In this study, we have developed a new photoacoustic endoscopic probe for targeting such applications and demonstrated its in vivo imaging ability through an animal experiment.

\section{MATERIALS AND METHODS}

\subsection{Photoacoustic endoscopic system}

The endoscopic probe is constructed with a rigid form for operator's more convenient handling during diagnostic procedures, and the probe body has a streamlined structure with a dome shaped distal end for smooth cavity introduction. Figure 1(a) shows a schematic of the endoscope. In the distal section, all optical and mechanical components are encapsulated in a medical-grade stainless steel tubular housing with an outer diameter of $\sim 12.7 \mathrm{~mm}$ and

${ }^{3}$ These authors contributed equally to this work. "Corresponding author: lhwang@biomed.wustl.edu

Photons Plus Ultrasound: Imaging and Sensing 2013, edited by Alexander A. Oraevsky, Lihong V. Wang, Proc. of SPIE Vol. 8581, 858148 - (C) 2013 SPIE · CCC code: 1605-7422/13/\$18 - doi: 10.1117/12.2005494 
$\sim 50 \mathrm{~cm}$ in length. The endoscope employs a parabolic shape scanning mirror actuated by a stepper motor located at the proximal end and performs a side scan, providing an angular field of view of $\sim 270^{\circ}$. This parabolic mirror was custommade, and it enables the acoustic focusing with a lower geometric aberration than our previous acoustic lens-based focusing methods ${ }^{2}$. The endoscope is comprised of the dome shape tubular stainless steel housing that encapsulates all elements, and a central tube that is statically located along axis of the endoscope and includes an optical fiber for light delivery, and a tubular rigid shaft that rotates between the two receiving the torque from the stepper motor.

(a)

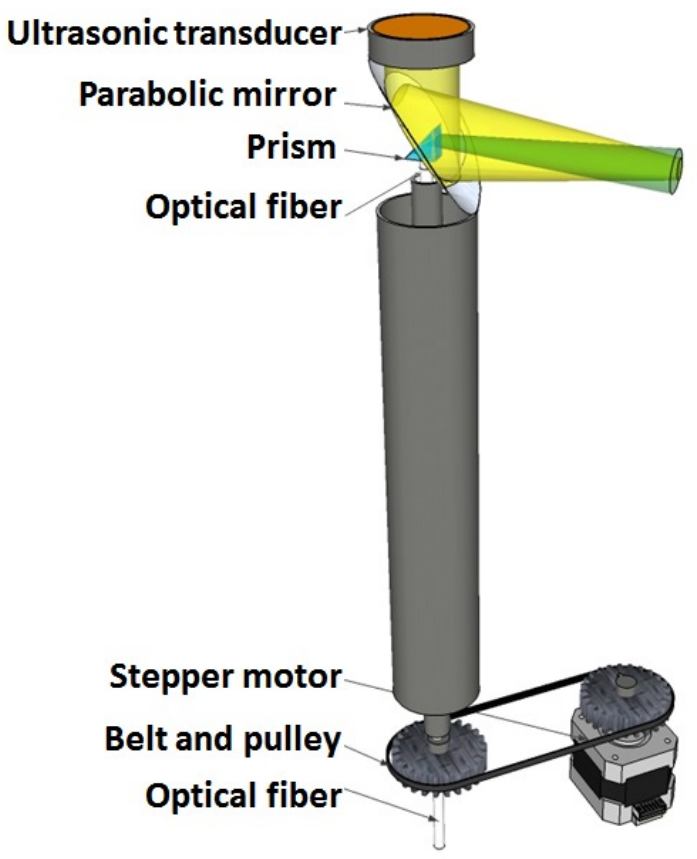

(b)

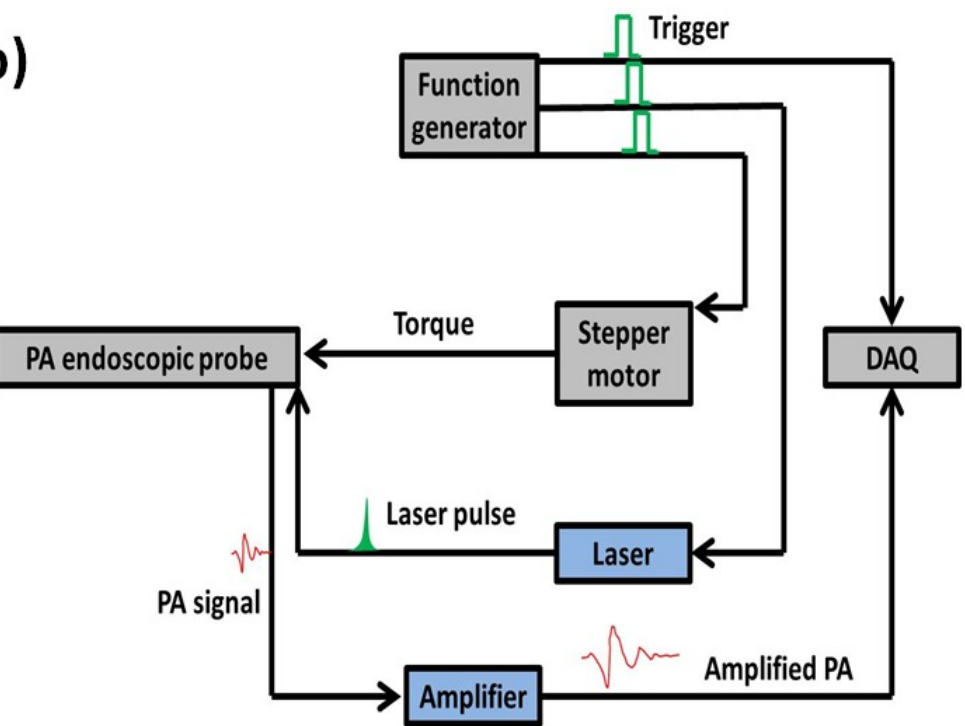

Figure 1. (a) Schematic of the endoscopic probe. (b) A diagram showing the entire endoscopic system. The stepper motor, laser system, and DAQ card are synchronized to the transistor-transistor logic (TTL) signals provided by the function generator.

Laser pulses exiting the optical fiber are reflected by an optical prism and then sent to the target tissue to generate PA signals. Some of the generated photoacoustic (PA) waves are then collected by the parabolic mirror, reflected, and 
finally detected by a flat ultrasonic transducer $\left(\sim 40 \mathrm{MHz}, \mathrm{LiNbO}_{3}\right)$. Figure 1(b) shows the endoscope's peripheral systems. For this endoscopic system, a function generator is used to drive the stepper motor, and to trigger the laser system and data acquisition (DAQ) card.

\subsection{System validation}

To quantify the endoscope's spatial resolution, we imaged a $\sim 20 \mu \mathrm{m}$ thick tungsten wire. We immersed the wire in a water tank together with the probe and placed the wire target at the focal position of the endoscope. We then performed $\mathrm{B}$-scan imaging in a plane perpendicular to the wire with an angular step size of $0.45^{\circ}$ for the scanning mirror, which yields an A-line number of 800 per B-scan. We recorded 100 B-scan images and averaged them for a more accurate measurement of resolutions.

The endoscope's in vivo imaging ability was also validated by imaging the abdomen of an adult Sprague Dawley rat ( $450 \mathrm{~g}$; Harlan National Customer Service Center) as a form of a handheld probe. After inducing an initial anesthesia with $\sim 4 \%$ isoflurane, we mounted the animal on a stage and maintained the anesthesia with $1.5-2.0 \%$ isoflurane supplied through a nose cone. We removed hair on the rat abdomen using hair removal lotion and spread ultrasound gel on the skin to provide acoustic coupling. Then, PA imaging was initiated using a laser wavelength of $523 \mathrm{~nm}(\sim 0.6$ $\mathrm{mJ} / \mathrm{pulse})$.

All procedures in the animal experiments followed the protocol approved by the Institutional Animal Care and Use Committee at Washington University in St. Louis.

\section{RESULTS}

\subsection{Resolution}

The resolution of the endoscopic system is determined by acoustics parameters. Since the imaged $\sim 20 \mu \mathrm{m}$ thick tungsten wire has a sufficiently length with a diameter smaller than the endoscope's spatial resolution, the wire can be treated as a line target in this resolution experiment. Figure 2 shows the acquired PA B-scan image that represents a line spread function of the system. We applied an envelope detection (Hilbert transformation) and finally determined a transverse resolution of $105 \mu \mathrm{m}$ and a radial resolution of $59 \mu \mathrm{m}$.

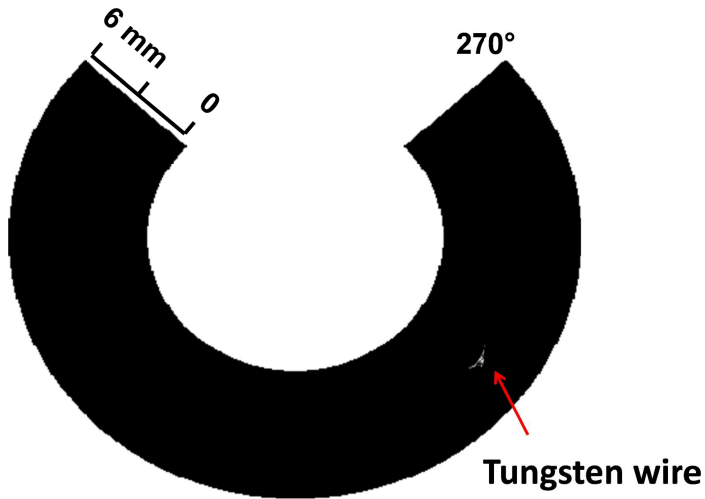

Figure 2. A representative PA image of a $20 \mu \mathrm{m}$ thick tungsten wire. This image shows the endoscope's angular field of view of covering $270^{\circ}$.

\subsection{Animal imaging results}

In Figure 3, we present three representative PA B-scan images acquired from the rat abdomen. As shown in these figures, PA signals mostly representing hemoglobin distribution were detected from a very large depth beneath the rat skin tissue, even from more than $5 \mathrm{~mm}$ depth. 
(a)
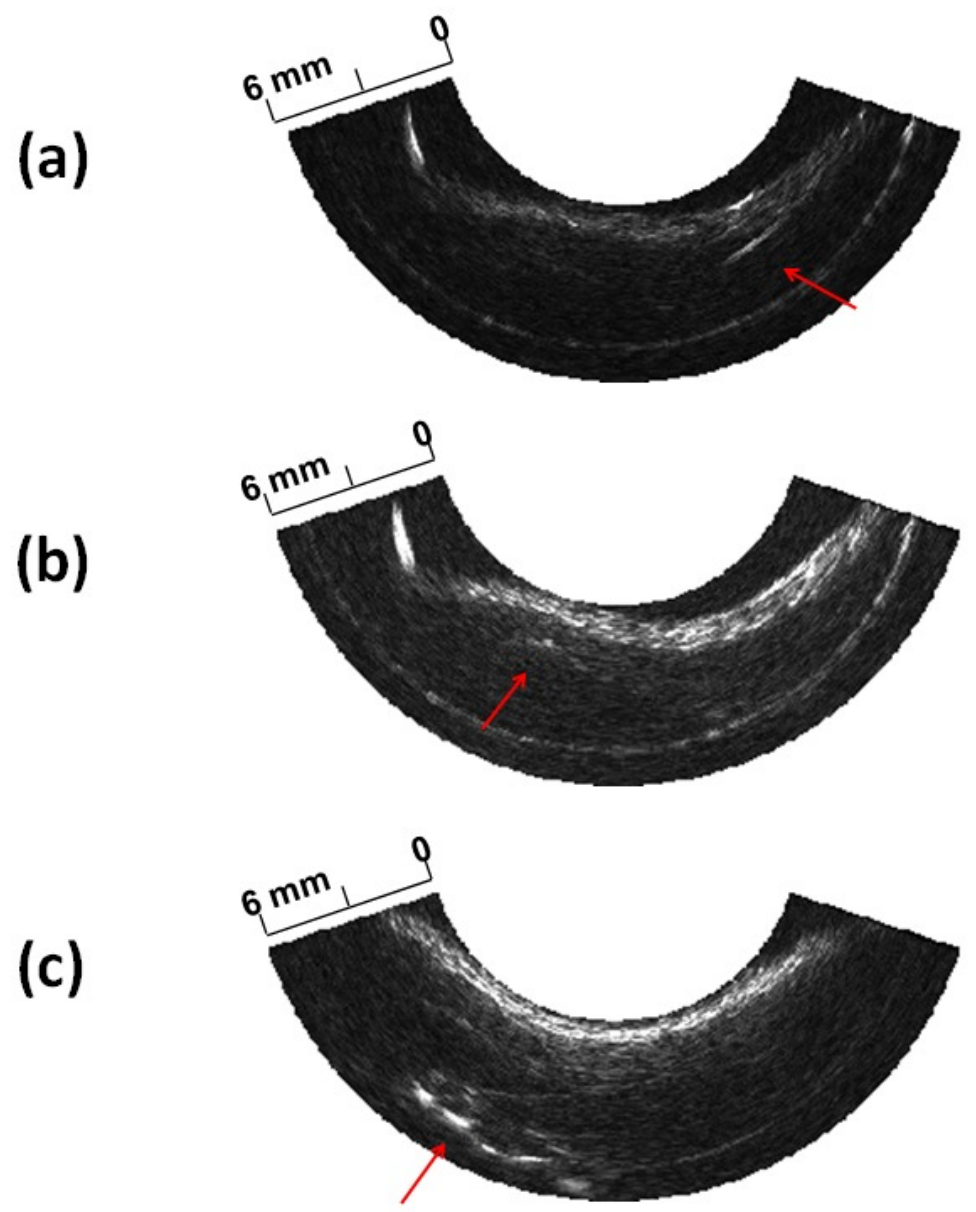

Figure 3. PA B-scan images acquired from a rat abdomen.

\section{DISCUSSION}

We have implemented a new PA endoscope with a design specifically targeted for human urogenital imaging application and validated the endoscope's imaging ability through a phantom and animal experiment. The endoscope is fully-encapsulated and it has a streamlined structure with a dome shaped distal end for smooth cavity introduction. Also, in forming the endoscope's housing, we utilized medical-grade stainless steels to comply with the material safety requirement. By coaxially aligning the optical illumination and the acoustic detection, we could achieve a more efficient overlapping of the two over a large radial range.

In contrast to our previous endoscopes that use micromotors, this endoscope adopts a proximal actuation mechanism; thereby the distal section's length could be shortened and the mechanical B-scan frame rate would potentially reach up to $30 \mathrm{~Hz}$, although the current imaging speed $(\sim 1 \mathrm{~Hz})$ is limited by the capacities of the employed DAQ card and laser system. Our next direction is to improve the DAQ part and to combine the endoscope with a compact laser system for use in clinical rooms. Also, we will enable volumetric imaging by adding a pullback scanning to the B-scan mechanism and also enable PA and US dual-mode imaging. 


\section{ACKNOWLEDGEMENT}

We thank Seema Dahlheimer for her attentive reading of the manuscript. This work was sponsored in part by National Institutes of Health grants R01 CA157277, R01 EB000712, R01 EB008085, R01 CA134539, U54 CA136398, R01 CA159959, and P41 EB002182. L.W. has a financial interest in Microphotoacoustics, Inc. and Endra, Inc., which, however, did not support this work.

\section{REFERENCES}

1. J. M. Yang, K. Maslov, H. C. Yang, Q. Zhou, K. K. Shung and L. V. Wang, "Photoacoustic endoscopy," Opt Lett 34(10), 1591-1593 (2009)

2. J. M. Yang, C. Favazza, R. Chen, J. Yao, K. Maslov, X. Cai, Q. Zhou, K. K. Shung and L. V. Wang, "Volumetric photoacoustic endoscopy of upper gastrointestinal tract: ultrasonic transducer technology development " Proc. SPIE 7899(78990D (2011)

3. J. M. Yang, C. Favazza, R. Chen, J. Yao, X. Cai, K. Maslov, Q. Zhou, K. K. Shung and L. V. Wang, "Simultaneous functional photoacoustic and ultrasonic endoscopy of internal organs in vivo," Nat Med 18(8), 1297-1302 (2012)

4. J. M. Yang, R. Chen, C. Favazza, J. Yao, C. Li, Z. Hu, Q. Zhou, K. K. Shung and L. V. Wang, "A 2.5-mm diameter probe for photoacoustic and ultrasonic endoscopy," Opt Express 20(21), 23944-23953 (2012)

5. L. V. Wang, "Multiscale photoacoustic microscopy and computed tomography," Nat Photonics 3(9), 503-509 (2009)

6. L. V. Wang and S. Hu, "Photoacoustic tomography: in vivo imaging from organelles to organs," Science 335(6075), 1458-1462 (2012)

7. Y. Yuan, S. Yang and D. Xing, "Preclinical photoacoustic imaging endoscope based on acousto-optic coaxial system using ring transducer array," Opt Lett 35(13), 2266-2268 (2010)

8. M. A. Yaseen, S. A. Ermilov, H. P. Brecht, R. Su, A. Conjusteau, M. Fronheiser, B. A. Bell, M. Motamedi and A. A. Oraevsky, "Optoacoustic imaging of the prostate: development toward image-guided biopsy," J Biomed Opt 15(2), $021310(2010)$

9. X. Wang, W. W. Roberts, P. L. Carson, D. P. Wood and J. B. Fowlkes, "Photoacoustic tomography: a potential new tool for prostate cancer," Biomed. Opt. Express 1(4), 1117-1126 (2010)

10. C. Sheaff and S. Ashkenazi, "A fiber optic optoacoustic ultrasound sensor for photoacoustic endoscopy," 2010 IEEE International Ultrasonics Symposium Proceedings 2135 - 2138 (2010)

11. Y. Yang, X. Li, T. Wang, P. D. Kumavor, A. Aguirre, K. K. Shung, Q. Zhou, M. Sanders, M. Brewer and Q. Zhu, "Integrated optical coherence tomography, ultrasound and photoacoustic imaging for ovarian tissue characterization," Biomed Opt Express 2(9), 2551-2561 (2011)

12. J. Yao, K. I. Maslov, Y. Zhang, Y. Xia and L. V. Wang, "Label-free oxygen-metabolic photoacoustic microscopy in vivo," J Biomed Opt 16(7), 076003 (2011)

13. Y. Wang, K. Maslov and L. V. Wang, "Spectrally encoded photoacoustic microscopy using a digital mirror device," J Biomed Opt 17(6), 066020 (2012)

14. J. Yao, C. H. Huang, L. Wang, J. M. Yang, L. Gao, K. I. Maslov, J. Zou and L. V. Wang, "Wide-field fast-scanning photoacoustic microscopy based on a water-immersible MEMS scanning mirror," J Biomed Opt 17(8), 080505$080501(2012)$

15. L. Gao, L. Wang, C. Li, Y. Liu, H. Ke, C. Zhang and L. V. Wang, "Single-cell photoacoustic thermometry," J Biomed Opt 18(2), 26003 (2013) 